DOI 10.35381/cm.v5i9.215

\title{
Cultura organizacional y su incidencia en el desempeño laboral de instituciones
} públicas gubernamentales

\section{Organizational culture and its impact on the job performance of government public institutions}

\author{
Victor Hugo Vásconez Samaniego \\ vvasconez@unach.edu.ec \\ Universidad Nacional de Chimborazo, Riobamba \\ Ecuador \\ https://orcid.org/0000-0003-2997-4768 \\ Henry David Vásconez Vásconez \\ hdvasconezv@hotmail.com \\ Escuela Superior Politécnica de Chimborazo, Riobamba \\ Ecuador \\ https://orcid.org/0000-0003-2648-3445 \\ Francisca Fernanda Guerrero Chávez \\ amantista fg@hotmail.com \\ Universidad Nacional de Chimborazo, Riobamba \\ Ecuador \\ https://orcid.org/0000-0002-2909-1271 \\ Luís Fernando Neira Altamirano \\ luis.neira@unach.edu.ec \\ Universidad Nacional de Chimborazo, Riobamba \\ Ecuador \\ https://orcid.org/0000-0002-4147-2501
}

Recibido: 25 de marzo de 2019

Aprobado: 1 de mayo de 2019 


\title{
RESUMEN
}

La investigación analiza la cultura organizacional y su incidencia en el desempeño laboral de la ciudad de Manta, Ecuador con la finalidad de conocer el desarrollo de las variables descritas y poder así, generar premisas que permitan contribuir al desarrollo de instituciones eficaces al servicio de la población, con lo cual se podrá velar por el respeto hacia la sociedad como principio humanizador. Se trabajó con un tipo de investigación descriptiva no experimental, la población estuvo conformada 171 funcionarios de instituciones públicas gubernamentales del Ecuador. La cultura organizacional incide en el desempeño laboral, en el caso de investigación con mayor incidencia sobre la perspectiva burocrática, siendo pertinente que la gestión pública realice una revisión de enfoque que le permita trascender en perspectiva a los nuevos modelos de administración pública.

Descriptores: Empresa pública; Administración pública; Cambio organizacional; Administración de empresas.

\begin{abstract}
The research analyzes the organizational culture and its impact on the work performance of the city of Manta, Ecuador with the purpose of knowing the development of the described variables and thus being able to generate premises that contribute to the development of effective institutions at the service of the population. , which will ensure respect for society as a humanizing principle. We worked with a type of non-experimental descriptive research, the population was made up of 171 officials from government public institutions in Ecuador. The organizational culture has an impact on work performance, in the case of research with a greater impact on the bureaucratic perspective, and it is pertinent for public management to carry out a review of the approach that allows it to transcend the new public administration models in perspective.
\end{abstract}

Descriptors: Public enterprises; Public administration; Organizational change; Business management.

\section{INTRODUCCIÓN}

En las organizaciones empresariales se han llevado unas series de cambios profundos e innovaciones que son cada vez más frecuentes debidos a los nuevos avances científicos, tecnológicos entre otros, por lo que las compañías se ven forzadas a adecuarse para estar acorde a las exigencias del mercado global, lo cual involucra 
cambios en el modo de cómo se adopta la cultura organizacional con la finalidad de ser organizaciones efectivas y exitosas.

La cultura organizacional sirve de marco de referencia a los miembros de la organización y da las pautas acerca de cómo las personas deben conducirse en ésta. En muchas ocasiones la cultura es tan evidente que se puede ver la conducta de la gente cambia en el momento en que traspasa las puertas de la empresa, podría decirse que las organizaciones comprometidas con el éxito, están abiertas a un constante aprendizaje y enmarcarlas como el activo fundamental de la organización. Sin embargo para que esto se pueda llevar a cabo, es necesario que el talento humano que forma parte de las organizaciones esté abierto a adaptarse a los cambios que se puedan dar en la empresa. Es importante destacar que el éxito empresarial depende ampliamente de la cultura organizacional y cómo influye está en el en el talento humano dentro de las organizaciones; siendo esta, la guía fundamental del comportamiento u actitud que deberían tener los empleados de la empresa en el momento en que ejercen sus funciones. Se destaca que la cultura organizacional es un conjunto dinámicos, valores, ideas, hábitos y tradiciones las cuales son compartidas por las personas que integran una empresa, por lo que es de mucha relevancia que esta influya de una manera positiva en el desempeño de las funciones ejercidas por los empleados de una organización.

Asimismo la cultura organizacional, es un aspecto importante que permite facilitar la comprensión de lo que realmente es la empresa, hacia donde se dirige y por su puesto cuales son los principios y valores por los cuales se rige la empresa. Es por ello que es de gran significado que todas las personas que laboren dentro de una organización se sientan comprometidas e identificadas con la cultura organizacional que se ejerza dentro de la empresa, esto con la finalidad de que el desempeño laboral del talento humano sea efectivo y por ende la calidad del servicio o producto ofrecido sea excelente. En efecto, el desempeño laboral según Chiavenato (2007):

En general es el esfuerzo de cada individuo está en función del valor de las recompensas ofrecidas y de la probabilidad de que éstas dependan del esfuerzo. Ese esfuerzo individual está dirigido, por una parte, por las 
capacidades y habilidades del individuo y, por otra, por las percepciones que él tiene del papel que debe desempeñar.

La interacción entre empleado y empresa es un proceso de reciprocidad, la organización realiza ciertas cosas por el trabajador y para el trabajador, lo remunera, le da seguridad y status; de modo recíproco, el empleado responde trabajando y desempeñando sus tareas, aunque el rendimiento que los empleados puedan tener dentro de la empresa, puede verse afectado o no dependiendo de cuan motivado este, por cuanto los empleados, están dispuestos a cooperar siempre y cuando sus actividades dentro de la organización, contribuyan directamente al logro de sus propios objetivos personales.

En este mismo orden y dirección, vale la pena resaltar que los trabajadores buscan cubrir ciertas expectativas en sus empleos tales como: reconocimiento por el trabajo realizado, sentir que participan en los procesos, estabilidad laboral, buenos salarios y buenas condiciones de trabajo; esto con la finalidad de que su desempeño sea óptimo.

Por tal razón resulta oportuno destacar que la cultura organizacional juega un papel fundamental en el desempeño profesional de los empleados dentro de las organizaciones, porque mientras estos se sientan más mezclados con la cultura organizacional de la misma ejercerán sus funciones de manera eficaz y por ende la productividad de la empresa mejorara. Así mismo Serna (2007) define la cultura organizacional resaltándola "como la manera en que las organizaciones hacen las cosas, establecen prioridades y dan importancia a las diferentes tareas empresariales".

Se puede observar que la cultura organizacional es el modo de ser de las empresas y la manera en que están efectúan sus funciones, enmarcadas en el compromiso de llevar a cabo sus tareas resaltando la cultura organizacional como su máximo exponente. Para Chiavenato (2007), la cultura organizacional es un "Modo de vida, un sistema de creencias, específicas y valores una forma particular de interacción y de rotación de determinada organización, cada organización es un sistema complejo y humano, que tiene características, cultura y sistemas de valores propios". 
Lo planteado por el autor, muestra que la cultura organizacional es un ente fundamental dentro de Las organizaciones, porque es la que permite la integración e interacción de todos los miembros, permitiéndoles sentirse identificados tanto con la misión, visión y valores propios de cada empresa y así mejorar su desempeño dentro de la empresa.

De esta manera la misión y visión de una empresa y los valores son elementos y ejes fundamentales que conforman una organización. Donde la misión es planteada por Thompson (2008), como:

El motivo, propósito, fin o razón de ser de la existencia de una empresa u organización porque define: 1) lo que pretende cumplir en su entorno o sistema social en el que actúa, 2) lo que pretende hacer, y 3) él para quién lo va a hacer; $y$ es influenciada en momentos concretos por algunos elementos como: la historia de la organización, las preferencias de la gerencia y/o de los propietarios, los factores externos o del entorno, los recursos disponibles, y sus capacidades distintivas (. p4).

Mientras que la visión no es más que "el camino al cual se dirige la empresa a largo plazo y sirve de rumbo y aliciente para orientar las decisiones estratégicas de crecimiento junto a las de competitividad" (Thompson 2008, p. 4). Por su parte los valores son herramientas que han venido ocupando un lugar cada vez más relevante en las teorías y prácticas de la administración en los últimos años. Cabe mencionar que los valores forman parte importante de una empresa ya que estos son un conjunto de principios, creencias, reglas que regulan la gestión de la organización. Constituyen la filosofía institucional y el soporte de la cultura organizacional.

Cada organización desarrolla un grupo central de suposiciones, conocimientos y reglas implícitas que gobiernan el conocimiento día a día en el lugar de trabajo; por lo que la cultura organizacional condiciona el comportamiento de las personas dentro de las organizaciones, es ello quien determina el recurso humano, además la cultura organizacional como un modo de vida, un sistema de creencias y valores, una forma aceptada de interacción y relaciones típicas de determinada organización.

Por otra parte a nivel latinoamericano, Vallenilla (2006) plantea que "Existe alguna evidencia que algunas organizaciones latinoamericanas han estado en transición, por 
ejemplo, han reportados cambios organizacionales, tales como el desarrollo de los recursos humanos y valores participativos entre otros"(p.26). Por lo que se puede observar que existe una preocupación latente por abocarse a comprender el campo del recurso humano, sin apartar los elementos de la cultura organizacional.

Desde lo planteado, la investigación analiza la cultura organizacional y su incidencia en el desempeño laboral de las instituciones públicas gubernamentales de la ciudad de Manta, Ecuador con la finalidad de conocer el desarrollo de las variables descritas y poder así, generar premisas que permitan contribuir al desarrollo de instituciones eficaces al servicio de la población, con lo cual se podrá velar por el respeto hacia la sociedad como principio humanizador, Aldana Zavala \& Isea (2018).

\section{METODOLOGÍA DE INVESTIGACIÓN}

La investigación fue tipo descriptiva, definida por Arias (2009) como la investigación que "consiste en la caracterización de un hecho, fenómeno o supo con establecer su estructura o comportamiento" (p. 64). Se abordó desde un diseño no experimental, transeccional de campo. Hernández, Fernández, Baptista (2014) refieren al diseño no experimental como "los estudios que se realizan sin la manipulación deliberada de variables y en los que sólo se observan los fenómenos en su ambiente natural para después analizarlos" (p. 267).

La población de acuerdo a Tamayo y Tamayo (2007), "Es la totalidad del fenómeno a estudiar donde las unidades de población poseen una característica común la cual se estudia y da origen a los datos de la investigación" ( $p$. 114), la cantidad de población estuvo conformada 171 funcionarios de instituciones públicas gubernamentales del Ecuador, a quienes se les aplicó un instrumento tipo escalamiento de Likert con la finalidad de conocer su percepción sobre las variables de estudio. 


\section{RESULTADOS}

Cultura burocrática

Cuadro 1

ALTERNATIVA

\begin{tabular}{lcc}
\hline Muy frecuentemente & 94 & 55 \\
Frecuentemente & 34 & 20 \\
Regularmente & 15 & 09 \\
Pocas veces & 18 & 10 \\
Nunca & 10 & 06 \\
\hline \multicolumn{1}{c}{ TOTAL } & 171 & 100,00
\end{tabular}

La alternativa muy frecuentemente tiene correspondencia de $55 \%$, la alternativa frecuentemente $20 \%$, regularmente $9 \%$, las alternativas pocas veces $10 \%$ y nunca $6 \%$

\begin{tabular}{|c|c|c|}
\hline $\begin{array}{l}\text { Cultura de clan } \\
\text { Cuadro } 2 \\
\qquad \text { ALTERNATIVA }\end{array}$ & FRECUENCIA (f) & PORCENTAJE \\
\hline Muy frecuentemente & 101 & 59 \\
\hline Frecuentemente & 27 & 16 \\
\hline Regularmente & 15 & 09 \\
\hline Pocas veces & 18 & 10 \\
\hline Nunca & 10 & 06 \\
\hline TOTAL & 171 & 100,00 \\
\hline
\end{tabular}

La alternativa muy frecuentemente tiene correspondencia de $59 \%$, la alternativa frecuentemente $16 \%$, regularmente $9 \%$, las alternativas pocas veces $10 \%$ y nunca $6 \%$ 


\section{Cultura emprendedora}

Cuadro 3

\begin{tabular}{lcc}
\multicolumn{1}{c}{ ALTERNATIVA } & FRECUENCIA (f) & PORCENTAJE \\
\hline Muy frecuentemente & 11 & 6 \\
Frecuentemente & 30 & 18 \\
Regularmente & 58 & 34 \\
Pocas veces & 27 & 16 \\
Nunca & 45 & 26 \\
\hline \multicolumn{1}{c}{ TOTAL } & 171 & 100,00
\end{tabular}

La alternativa muy frecuentemente tiene correspondencia de $6 \%$, la alternativa frecuentemente $18 \%$, regularmente $34 \%$, las alternativas pocas veces $16 \%$ y nunca $26 \%$

\section{Cultura de mercado}

Cuadro 4

\begin{tabular}{lcc}
$\quad$ ALTERNATIVA & FRECUENCIA (f) & PORCENTAJE \\
\hline Muy frecuentemente & 19 & 11 \\
Frecuentemente & 21 & 12 \\
Regularmente & 56 & 33 \\
Pocas veces & 15 & 15 \\
Nunca & 29 & 29 \\
\hline \multicolumn{1}{c}{ TOTAL } & 171 & 100,00
\end{tabular}

La alternativa muy frecuentemente tiene correspondencia de $11 \%$, la alternativa frecuentemente $12 \%$, regularmente $33 \%$, las alternativas pocas veces $15 \%$ y nunca $29 \%$ 
Desempeño laboral

Cuadro 5

\begin{tabular}{|c|c|c|}
\hline ALTERNATIVA & $\begin{array}{l}\text { FRECUENCIA } \\
\text { (f) }\end{array}$ & PORCENTAJE \\
\hline $\begin{array}{l}\text { Rendimiento de la tarea específica del } \\
\text { puesto }\end{array}$ & 91 & 53 \\
\hline $\begin{array}{l}\text { Rendimiento de la tarea no especifica } \\
\text { del puesto }\end{array}$ & 54 & 32 \\
\hline Demostración del esfuerzo & 21 & 12 \\
\hline Mantenimiento de la disciplina personal & 05 & 03 \\
\hline TOTAL & 171 & 100,00 \\
\hline
\end{tabular}

\section{DISCUSIÓN}

La tendencia de respuestas en cultura burocrática y cultura de clan, tienden hacia las opciones de respuesta positiva, lo cual indica que es una institución donde se cuida el formalismo de los procedimientos establecidos en los protocolos, manuales, para efectuar un determinado trabajo, así mismo se evidencia un establecimiento de la cultura del clan, generándose que los empleados de mayor antigüedad tienen incidencia en las decisiones que toman los de menos tiempo o rango, lo cual concuerda con los aspectos teóricos planteados por Hellriegel Don/ Slucum John (2009). 
En cuanto a la cultura emprendedora y cultura de mercado, la tendencia porcentual se inclina hacia las opciones negativas, se evidencia que los empleados no toman decisiones que impliquen riesgos, innovación, en el desarrollo del trabajo que generan, así como tampoco tienen una alta valoración de compromiso con el cliente externo, lo cual se distancia de lo propuesto por Hellriegel Don/ Slucum John (2009).

En cuanto el desempeño laboral, se evidencia que los empleados tienen mayor efectividad desde el rendimiento de la tarea no específica del puesto, lo cual desde la visión de Campbell (1990), señala que esto obedece cuando el empleado se guía por estructuras preestablecidas en la organización, lo cual le permite desarrollar un trabajo apegado a lo establecido en el manual.

\section{CONCLUSIONES}

La población de estudio, se caracteriza por tener una cultura organizacional con mayor énfasis en lo burocrático, lo cual impide que se genere una aptitud de emprendimiento e innovación, siendo esto distante de lo establecido en los enfoques de la nueva administración o gestión pública, donde se procura que estas actúen en similitud de la empresa privada, para lo cual se requiere prestar un servicio de calidad al cliente, aunado a la generación de posibilidades de promover el crecimiento continuo de la organización. Para transcender lo planteado hacia una visión basada en nuevas tendencias administrativas, es necesario a juicio de León (2017), generar una trasposición organizacional que permita por medio de la reflexión generada por medio de metáforas, construir nuevas estructuras organizativas en donde el talento humano se percibe como el motor central de la empresa, teniendo en cuenta que su participación activa es fundamental para lograr el éxito colectivo de la organización.

Aunado a lo planteado, es pertinente que las organizaciones públicas, transiten hacia la consolidación de una empresa basada en lo sistémico con la finalidad de generar nuevas visiones de liderazgo que permitan impulsar hacia un trabajo cooperativo en función de lograr los propósitos en la gestión organizacional de la empresa, tal como señalan Aldana, 
Salón \& Guzmán (2019). De ese modo, se reconoce que la cultura organizacional incide en el desempeño laboral, en el caso de investigación con mayor incidencia sobre la perspectiva burocrática, siendo pertinente que la gestión pública realice una revisión de enfoque que le permita trascender en perspectiva a los nuevos modelos de administración pública.

\section{REFERENCIAS CONSULTADAS}

1. Aldana, J., Salón, M., \& Guzmán, N. (2019). Liderazgo sistémico en las competencias gerenciales docentes universitarias. CIENCIAMATRIA, 5(8), 50-74. Recuperado a partir de http://cienciamatriarevista.org.ve/index.php/cm/article/view/87

2. Aldana Zavala, J., \& Isea, J. (2018). Derechos Humanos y Dignidad Humana. IUSTITIA SOCIALIS, 3(4), 8-23. Recuperado de http://fundacionkoinonia.com.ve/ojs/index.php/lustitia Socialis/article/view/119/10 1

3. Arias, F. (2009). El Proyecto de Investigación. Guía para su elaboración. Caracas: Epísteme. Quinta Edición.

4. Campbell, J. P. (1990). Modeling theperformance prediction problem in indus-trial and organizational psychology. En:M. D. Dunnette y L. M. Hough (Eds):Handbook of industrial and organization-al psychology, Vol. 1 ( $2^{\mathrm{a}} \mathrm{ed}$.) (pp. 687-732). Palo Alto, CA, US: Consulting Psy-chologists Press.

5. Chiavenato, I. (2007). Administración de los Recursos Humanos. (7ma. Edición). Santa Fe de Bogotá, Colombia: Editorial Mc Graw-Hill.

6. Hernández, Fernández y Baptista (2014). Metodología de la investigación. México, Mc Graw Hill Hispanoamericana. Hill Internacional.

7. Hellriegel Don/ Slucum John (2009). Comportamiento Organizacional. Mc Graw Hill. México.

8. León, F. (2017). Transposición de saberes desde el análisis organizacional de las metáforas de Morgan: herramienta para romper paradigmas de la gerencia académica universitaria en Venezuela. Revista Arbitrada Interdisciplinaria Koinonía, 2(3), 31-47. Recuperado de http://fundacionkoinonia.com.ve/ojs/index.php/revistakoinonia/article/view/50/37 
9. Serna H, (2007). Gerencia Estratégica. Colombia: 3r Editores .Ltd

10. Tamayo y Tamayo (2009). El proceso de investigación científica. Limusa. México.

11. Thompson Arthur y Strickland A. J. (2008). Administración Estratégica Conceptos y Casos. Mc Graw Hill, México.

12. Vallenilla, F. (2006). Cultura Organizacional y Posmodernidad: Una aproximación al caso Venezuela. Revista NEGOTIUM de Ciencias Gerenciales. Año 1 Nro. 3.

\section{REFERENCIAS CONSULTADAS}

1. Aldana, J., Salón, M., \& Guzmán, N. (2019). Systemic leadership in university teaching management skills. SCIENCE, 5 (8), 50-74. Recovered from http://cienciamatriarevista.org.ve/index.php/cm/article/view/87

2. Aldana Zavala, J., \& Isea, J. (2018). Human Rights and Human Dignity. IUSTITIA SOCIALIS, $\quad 3 \quad$ (4), 8-23. $\quad$ Retrieved from http://fundacionkoinonia.com.ve/ojs/index.php/lustitia Socialis/article/view/119/10 1

3. Arias, F. (2009). The Research Project Guide for its elaboration. Caracas: Epistle me. Fifth edition.

4. Campbell, J. P. (1990). Modeling theperformance prediction problem in indus-trial and organizational psychology. In: M. D. Dunnette and L. M. Hough (Eds): Handbook of industrial and organization-al psychology, Vol. 1 (2nd ed.) (Pp. 687732). Palo Alto, CA, US: Consulting Psy-chologists Press.

5. Chiavenato, I. (2007). Human Resources Administration. (7th Edition). Santa Fe de Bogotá, Colombia: Mc Graw-Hill Publishing House.

6. Hernández, Fernández and Baptista (2014). Investigation methodology. Mexico, Mc Graw Hill Hispanic American. Hill International

7. Hellriegel Don / Slucum John (2009). Organizational behavior. Mc Graw Hill Mexico. 
Luis Fernando Neira Altamirano; Henry David Vásconez Vásconez; Victor Hugo Vásconez Samaniego; Francisca Fernanda Guerrero Chávez

8. León, F. (2017). Transposition of knowledge from the organizational analysis of Morgan's metaphors: a tool to break paradigms of university academic management in Venezuela. Interdisciplinary Arbitrated Review Koinonía, 2 (3), 31 47. http://fundacionkoinonia.com.ve/ojs/index.php/revistakoinonia/article/view/50/37

9. Serna H, (2007). Strategic management. Colombia: 3rd Editors .Ltd

10. Tamayo and Tamayo (2009). The process of scientific research. Limusa Mexico.

11. Thompson Arthur and Strickland A. J. (2008). Strategic Administration Concepts and Cases. Mc Graw Hill, Mexico.

12. Vallenilla, F. (2006). Organizational Culture and Postmodernity: An approach to the Venezuela case. NEGOTIUM Magazine of Management Sciences. Year 1 No. 3.

(C2019 por los autores. Este artículo es de acceso abierto y distribuido según los términos y condiciones de la licencia Creative Commons Atribución-NoComercial-Compartirlgual 4.0 Internacional (CC BY-NC-

SA 4.0) (https://creativecommons.org/licenses/by-nc-sa/4.0/). 